\title{
Threading an elephant through the eye of a needle: Where are platelets made?
}

Cell Research (2017) 27:1079-1080. doi:10.1038/cr.2017.65; published online 9 May 2017

There has been a long-standing controversy of whether megakaryocytes release platelets in the marrow or travel to the lungs and release platelets there. Using two-photon electron microscopy and orthotopic lung transplantation, Lefrançais et al. now document that platelet release occurs physiologically in the lungs of mice from extrapulmonary megakaryocytes and that this release accounts for $\sim \mathbf{5 0} \%$ of total platelet production.

"Histological examination of the lungs with a technique adequate to give a differential staining of platelet material demonstrates the presence of giant cells in the lungs and supports the view that they are active in the production of platelets." William Howell, 1937

In mammals, it is ironic that platelets - small, anucleate cellular fragments key to normal hemostasis - arise from large, polyploid cells termed megakaryocytes. Platelet release or thrombopoiesis had been assumed to occur in adults within the bone marrow where megakaryocytes develop (Figure 1A). The released platelets subsequently reach the circulation. Initial direct visualization of the marrow with twophoton excitation microscopy (TPEM) was interpreted to show that platelets were instead released as megakaryocytes traversed from the intramedullar space into the circulation [1] (Figure 1B), relegating intramedullar release to "stress" thrombopoiesis [2]. However, these same videos showed an occasional whole megakaryocyte extruding from the marrow. Further, most of these and additional videos [3] did not show platelet release from megakaryocytes. Instead, the megakaryocytes extruded a single process through the endothelial lining and released large cytoplasmic fragments that were often clearly attached as beads on a string (Figure 1C). These videos are consistent with much earlier observations including Aschoff's 1893 observation of intravascular "giant cells" in the lungs [4]. In 1937, Howell et al., as quoted above, proposed that these cells release platelets in the lung with platelet counts higher in the pulmonary veins than arteries [5]. Based on this alternative model, Fuentes et al. [6] showed that infused murine megakaryocytes are transiently entrapped in the lungs of recipient mice and release a wave of new platelets, supporting that pulmonary megakaryocyte entrapment could potentially be a mechanism for releasing platelets.

Now, Lefrançais et al. [7] provide the first direct visualization that native megakaryocytes are entrapped in the lungs, each releasing a shower of 500-1 000 platelets over 20-60 min using high quality TPEM and a series of megakaryocyte-specific reporter markers (Figure 1C). By assigning surface volumes to the observed megakaryocytes and extrapolating to the entire lung volume, the authors estimated that $\sim 50 \%$ of platelet production in the mouse can be attributed to pulmonary thrombopoiesis. Orthotopic lung transplants between mice confirmed that entrapped intravascular megakaryocytes were of extrapulmonary origin. In addition, the authors identified a second important and perhaps independent set of observations based on finding primi- tive megakaryocytes and hematopoietic progenitors in the extravascular space of the lungs. When lungs were transplanted to thrombocytopenic or hematopoietic stem cell-deficient animals, these cells were able to reconstitute platelet counts and contribute to multi-lineage hematopoiesis.

From a biological perspective, the improbable scenario that giant megakaryocytes extricate themselves from the marrow and travel intactly to the lungs to release platelets appears to be correct at least for mice. Whether it occurs in humans needs direct examination, but we do know that infused human megakaryocytes into recipient mice are entrapped in the lungs and release a wave of normal-appearing platelets over a similar timeframe as in the Lefrançais paper [8]. Additionally, patients with right-to-left pulmonary shunts have low platelet counts that have been attributed to the role of the pulmonary vascular bed in thrombopoiesis [9]. Clearly, the shoe is now on the other foot: does any thrombopoiesis actually occur in the marrow (Figure 1A) or at its vascular border (Figure 1B)?

The Lefrançais paper also raises many new questions including what are the features of the marrow and pulmonary vascular linings that enhance this marrow/pulmonary axis? Is megakaryocyte transmigration across the marrow lining due to the endothelium's previously described fenestrations? Is the medullar-to-vascular sphingosine1-phosphate gradient [3] sufficient for megakaryocyte transmigration? Are there also peculiar features of the pulmonary vascular bed that makes it 


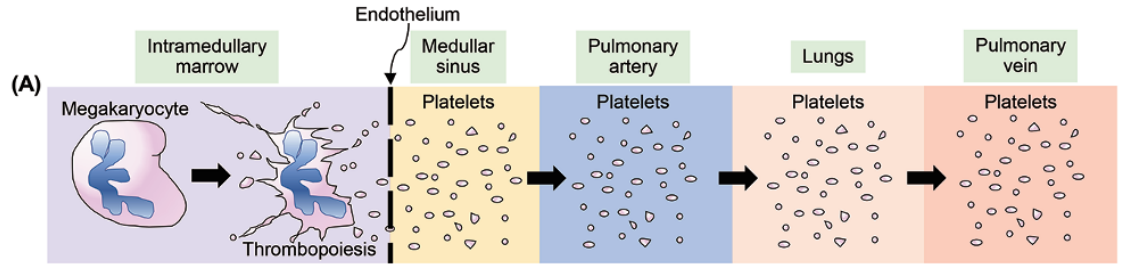

(B)

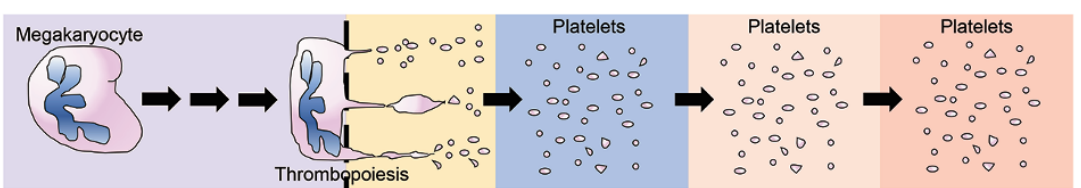

(C)

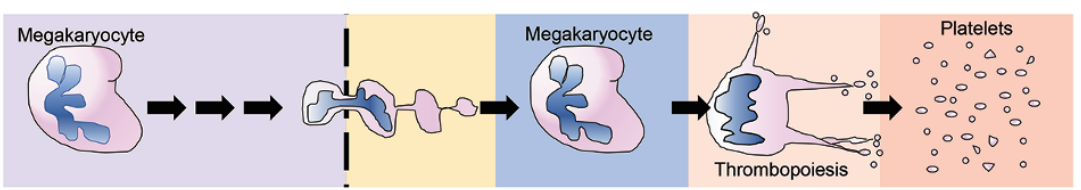

Figure 1 Alternative models for the site of thrombopoiesis. (A-C) Schematics showing from left to right, the intramedullary marrow, the fenestrated endothelial lining separating the marrow from the medullar sinuses, the medullar sinus, which through the returning veins (not shown), connects to the pulmonary arteries, followed by the pulmonary microcirculation (lungs) and then the pulmonary veins prior to emptying into the general circulation (not shown). Intramedullar release of platelets (A). Release of platelets as megakaryocytes traverse the medullar endothelial lining (B). Pulmonary release of platelets from megakaryocytes that have traveled from the marrow as supported by Lefrançais et al. (C).

conducive to thrombopoiesis or is it just the first vascular bed encountered? Lefrançais et al. point out that pulmonary arteries are rich in von Willebrand Factor, but patients with Type 3 severe von Willibrand Disease do not have thrombocytopenia. Whether a particular ligand/receptor system or the peculiar design of the pulmonary microvasculature or its marked oxygen and $\mathrm{pH}$ gradients are essential for the observed thrombopoiesis is unknown.

The lung as a hematopoietic organ is also intriguing. In both mice and humans, the fetal liver is the major site of hematopoiesis before the marrow takes over in adults, but only mice undergo hematopoiesis in the spleen. Whether the hematopoietic findings in murine lungs are species-specific now requires study. When marrow is infused into patients during marrow transplantation, cells first home to the lungs before trafficking to the marrow. Perhaps Lefrançais et al. have defined a way station during the extramedullary passage of hematopoietic progenitor cells? Whether the lung is a unique organ in this regard requires further study.

From a clinical perspective, findings by Lefrançais et al. raise questions of the implications of pulmonary thrombopoiesis in various clinical settings. There are an excess number of pulmonary capillaries so that only $1 \%-2 \%$ of them are obstructed by megakaryocytes during a portion of each day. What happens if this excess disappears for example in emphysema? Can megakaryocytes obstruct enough capillaries to have a clinical impact? Immune thrombocytopenia purpura is another pathologic state that needs revisiting. What happens to megakaryocytes coated with pathogenic antibodies traveling to and then entrapped in the lungs? Does the number of entrapped megakaryocytes decrease? Is their ability to undergo thrombopoiesis reduced?

Finally, there has been a great deal of research interest in developing platelet transfusions from in vitro-grown megakaryocytes. Shed "platelets" in culture are problematic [8]. A series of platelet bioreactors have been designed mostly based on Figure 1B's model of platelet release. These have yet to yield a population of platelets comparable to donor-derived platelets [10]. Better insights into how the pulmonary vasculature contributes to platelet release might lead to better designed bioreactors. Alternatively, demonstration by Lefrançais et al. that megakaryocytes physiologically travel to the lungs may lead to direct infusions of mature megakaryocytes into patients, allowing the multi-talented lung to do its magic. Infusions in mice with either mouse or human megakaryocytes have yielded released platelets functionally very similar to donor-derived platelets $[6,8]$. The Lefrançais paper provides support for developing this approach for clinical application.

Ian Johnston ${ }^{1}$, Vincent Hayes ${ }^{2}$,
Mortimer Poncz

${ }^{1}$ Department of Pharmacology, ${ }^{2}$ Department of Pathology, ${ }^{3}$ Department of Pediatrics, The Perelman School of Medicine at the University of Pennsylvania, Philadelphia, PA, USA; ${ }^{4}$ Division of Pediatrics, The Children's Hospital of Philadelphia, 3615 Civic Center Blvd, Philadelphia, PA 19104, USA

Correspondence: Mortimer Poncz

Tel: +1-610-888-7442

E-mail: poncz@email.chop.edu

\section{References}

1 Junt $\mathrm{T}$, Schulze $\mathrm{H}$, Chen Z, et al. Science 2007; 317:1767-1770.

2 Nishimura S, Nagasaki M, Kunishima S, et al. J Cell Biol 2015; 209:453-466.

3 Zhang L, Orban M, Lorenz M, et al. J Exp Med 2012; 209:2165-2181.

4 Aschoff L. Arch Pathol Anat Phys 1893; 134:11-14

5 Howell WH, Donahue DD. J Exp Med 1937; 65:177-203.

6 Fuentes R, Wang Y, Hirsch J, et al. J Clin Invest 2010; 120:3917-3922.

7 Lefrançais E, Ortiz-Muñoz G, Caudrillier A, et al. Nature 2017; 544:105-109.

8 Wang Y, Hayes V, Jarocha D, et al. Blood 2015; 125:3627-3636.

9 Lill MC, Perloff JK, Child JS. Am J Cardiol 2006; 98:254-258.

10 Sim X, Poncz M, Gadue P, et al. Blood 2016; 127:1227-1233. 